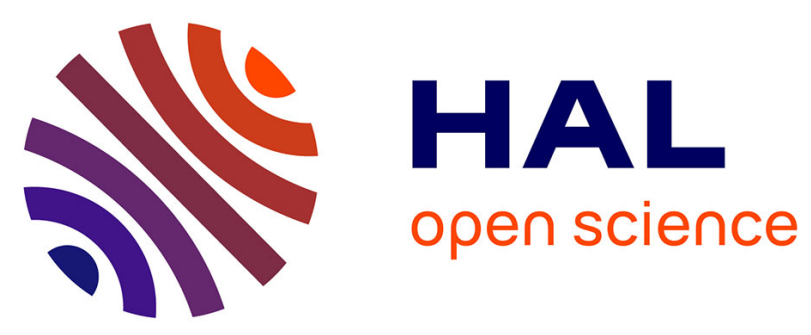

\title{
Adaptive Parameterized Consistency for Non-Binary CSPs by Counting Supports
}

Robert J. Woodward, Anthony Schneider, Berthe Y. Choueiry, Christian Bessiere

\section{- To cite this version:}

Robert J. Woodward, Anthony Schneider, Berthe Y. Choueiry, Christian Bessiere. Adaptive Parameterized Consistency for Non-Binary CSPs by Counting Supports. CP: Principles and Practice of Constraint Programming, Sep 2014, Lyon, France. pp.755-764, 10.1007/978-3-319-10428-7_54 . lirmm-01067342

\section{HAL Id: lirmm-01067342 https://hal-lirmm.ccsd.cnrs.fr/lirmm-01067342}

Submitted on 10 Oct 2019

HAL is a multi-disciplinary open access archive for the deposit and dissemination of scientific research documents, whether they are published or not. The documents may come from teaching and research institutions in France or abroad, or from public or private research centers.
L'archive ouverte pluridisciplinaire HAL, est destinée au dépôt et à la diffusion de documents scientifiques de niveau recherche, publiés ou non, émanant des établissements d'enseignement et de recherche français ou étrangers, des laboratoires publics ou privés. 


\title{
Adaptive Parameterized Consistency for Non-Binary CSPs by Counting Supports ${ }^{\star}$
}

\author{
Robert J. Woodward ${ }^{1,2}$, Anthony Schneider ${ }^{1}$, Berthe Y. Choueiry ${ }^{1}$, and \\ Christian Bessiere ${ }^{2}$ \\ 1 Constraint Systems Laboratory, University of Nebraska-Lincoln, USA \\ \{rwoodwar|aschneid|choueiry\}@cse.unl.edu \\ 2 CNRS, University of Montpellier, France \\ bessiere@lirmm.fr
}

\begin{abstract}
Determining the appropriate level of local consistency to enforce on a given instance of a Constraint Satisfaction Problem (CSP) is not an easy task. However, selecting the right level may determine our ability to solve the problem. Adaptive parameterized consistency was recently proposed for binary CSPs as a strategy to dynamically select one of two local consistencies (i.e., $\mathrm{AC}$ and maxRPC). In this paper, we propose a similar strategy for non-binary table constraints to select between enforcing GAC and pairwise consistency. While the former strategy approximates the supports by their rank and requires that the variables domains be ordered, our technique removes those limitations. We empirically evaluate our approach on benchmark problems to establish its advantages.
\end{abstract}

\section{Introduction}

There is an abundance of local consistency techniques of varying cost and pruning power to apply to a Constraint Satisfaction Problem (CSP), but choosing the right one for a given instance remains an open question. In a portfolio approach $[22,11,7]$, we typically choose a single consistency level and enforce it on the entire problem (or a subproblem). Heuristic-based methods have been proposed to dynamically switch, at various stages of search and depending on the constraint, between a weak and a strong level of consistency, AC and maxRPC for binary CSPs [20] and GAC and maxRPWC for non-binary CSPs [18]. The above-mentioned approaches do not allow us to enforce different levels of consistency on the values in the domain of the same variable. To this end, Balafrej et al. introduced adaptive parameterized consistency, which selects, for each value in the domain of a variable, one of two consistency levels based on the value of a parameter [1]. That parameter is determined by the rank of the support of the value in a constraint (assuming a fixed total ordering of the variables' domains),

\footnotetext{
* This research was supported by NSF Grant No. RI-111795 and EU project ICON (FP7-284715). Woodward was supported by an NSF GRF Grant No. 1041000 and a Chateaubriand Fellowship. Experiments were conducted on the equipment of the Holland Computing Center at the University of Nebraska-Lincoln.
} 
and updated depending on the weight of the constraint [5]. Their study targeted enforcing $\mathrm{AC}$ and maxRPC on binary CSPs.

In this paper, we extend their mechanism to enforcing GAC and pairwiseconsistency on non-binary CSPs with table constraints. Our approach is based on counting the number of supporting tuples, which is automatically provided by the algorithms that we use. Thus, we remove the restriction on maintaining ordered domains and the approximation of a support's count by its rank. We establish empirically the advantages of our approach.

The paper is structured as follows. Section 2 provides background information. Section 3 describes our approach, and Section 4 discusses our empirically evaluation on benchmark problems. Finally, Section 5 concludes the paper.

\section{Background}

We first summarize the main concepts and definitions used.

\subsection{Constraint Satisfaction Problem}

A Constraint Satisfaction Problem (CSP) is defined by a tuple $(\mathcal{X}, \mathcal{D}, \mathcal{C})$, where $\mathcal{X}$ is a set of variables, $\mathcal{D}$ is a set of domains, and $\mathcal{C}$ is a set of constraints. Each variable $x_{i} \in \mathcal{X}$ is associated a finite domain $\operatorname{dom}\left(x_{i}\right) \in \mathcal{D}$. We denote a variablevalue pair as $\left\langle x_{i}, v_{i}\right\rangle$, where $v_{i} \in \operatorname{dom}\left(x_{i}\right)$. Each constraint $c_{j} \in \mathcal{C}$ is defined in extension by a relation $R_{j}$ specified over the scope of the constraint, scope $\left(c_{j}\right)$, which is the set of variables to which the constraint applies. For readability, we refer to the scope of a relation $\operatorname{scope}\left(R_{j}\right)=\operatorname{scope}\left(c_{j}\right)$. A tuple $\tau \in R_{j}$ is a combination of allowed values for the variables in $\operatorname{scope}\left(R_{j}\right) . \tau\left[x_{i}\right]$ is the value that the variable $x_{i}$ takes in $\tau$. We denote cons $\left(x_{i}\right)$ as the set of constraints that apply to variable $x_{i}$, and neigh $\left(c_{j}\right)$ the set of constraints whose scopes overlap with $c_{j}$. When $\left|\operatorname{scope}\left(c_{j}\right)\right|=2, c_{j}$ is said to be a binary constraint, otherwise, it is non-binary. A solution to the CSP assigns, to each variable, a value taken from its domain such that all the constraints are satisfied. Deciding the existence of a solution for a CSP is NP-complete.

\subsection{Local Consistency Properties}

CSPs are typically solved with backtrack search. To reduce the severity of the combinatorial explosion, CSPs are usually filtered by enforcing a given local consistency property [2].

A variable-value pair $\left\langle x_{i}, v_{i}\right\rangle$ has an arc-consistent support (AC-support) $\left\langle x_{j}, v_{j}\right\rangle$ if the tuple $\left(v_{i}, v_{j}\right) \in R_{i j}$ where $\operatorname{scope}\left(R_{i j}\right)=\left\{x_{i}, x_{j}\right\}[16,3]$. A CSP is arc consistent if every variable-value pair has an AC-support in every constraint. Generalized Arc Consistency (GAC) generalizes arc consistency to non-binary CSPs [16]. $\left\langle x_{i}, v_{i}\right\rangle$ has a GAC-support in constraint $c_{j}$ if $\exists \tau \in R_{j}$ such that $\tau\left[x_{i}\right]=v_{i}$. A CSP is GAC if every $\left\langle x_{i}, v_{i}\right\rangle$ has a GAC-support in every constraint in cons $\left(x_{i}\right)$. GAC can be enforced by removing domain values that have no GAC-support, leaving the relations unchanged. Simple Tabular Reduction (STR) algorithms not only enforce GAC on the domains, but also remove all tuples $\tau \in R_{j}$ where $\exists x_{i} \in \operatorname{scope}\left(R_{j}\right)$ such that $\tau\left[x_{i}\right] \notin \operatorname{dom}\left(x_{i}\right)[21,13,14]$. 
A CSP is $m$-wise consistent if, every tuple in a relation can be extended to every combination of $m-1$ other relations in a consistent manner [8, 10]. Keeping with relational-consistency notations, Karakashian et al. denoted $m$-wise consistency by $\mathrm{R}(*, m) \mathrm{C}$, and proposed a first algorithm for enforcing it [12]. Their implementation finds an extension (i.e., support) for a tuple by conducting a backtrack search on the other $m-1$ relations, and removes the tuples that have no support. After all relations are filtered, they are projected onto the domains of the variables. Pairwise consistency (PWC) corresponds to $m=2, \mathrm{R}(*, 2) \mathrm{C} \equiv \mathrm{PWC}$. Lecoutre et al. introduced the algorithm extended STR (eSTR) [15], which enforces PWC on a CSP using the STR mechanism [21]. eSTR maintains counters on the intersections of two constraints to determine if a tuple is pairwise consistent or not. In this paper, we enforce PWC using the algorithm for $\mathrm{R}(*, 2) \mathrm{C}[12]$, and not eSTR, because it is prohibitively expensive to continuously maintain the counters of eSTR in a strategy where PWC is only selectively enforced.

\subsection{Adaptive Parameterized Consistency}

Balafrej et al. introduced the distance to the end of value $v_{i}$ for variable $x_{i}$ as:

$$
\Delta\left(x_{i}, v_{i}\right)=\frac{\left|\operatorname{dom}^{o}\left(x_{i}\right)\right|-\operatorname{rank}\left(v_{i}, \operatorname{dom}^{o}\left(x_{i}\right)\right)}{\left|\operatorname{dom}^{o}\left(x_{i}\right)\right|}
$$

where $\operatorname{dom}^{o}\left(x_{i}\right)$ is the original, unfiltered domain of $x_{i}$, and $\operatorname{rank}\left(v_{i}, \operatorname{dom}^{o}\left(x_{i}\right)\right)$ is the position of $v_{i}$ in the ordered set $\operatorname{dom}^{o}\left(x_{i}\right)$ [1]. In Figure 1, borrowed from [1], $\Delta\left(x_{2}, 1\right)=0.75, \Delta\left(x_{2}, 2\right)=0.50, \Delta\left(x_{2}, 3\right)=0.25$, and $\Delta\left(x_{2}, 4\right)=0.00$.

Further, for a given parameter $p$, they defined $\left\langle x_{i}, v_{i}\right\rangle$ to be $p$-stable for $A C$ for $c_{i j}$ where $\operatorname{scope}\left(c_{i j}\right)=\left\{x_{i}, x_{j}\right\}$ if there exists an AC-support $\left\langle x_{j}, v_{j}\right\rangle$ with $\Delta\left(x_{j}, v_{j}\right) \geq p$ for $c_{i j}$. Figure 1 illustrates an example for the constraint $x_{1} \leq x_{2}$ with $p=0.25$. $\left\langle x_{1}, 1\right\rangle,\left\langle x_{1}, 2\right\rangle,\left\langle x_{1}, 3\right\rangle$ are all 0.25 -stable for AC for the constraint, but $\left\langle x_{1}, 4\right\rangle$ is not, because its only AC-support, $\left\langle x_{2}, 4\right\rangle$, has distance 0 .

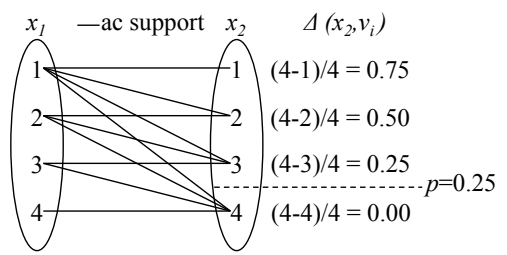

Fig. 1. The constraint $x_{1} \leq x_{2} .\left\langle x_{1}, 4\right\rangle$ is not 0.25 -stable for AC [1].

The parameterized strategy $p$-LC [1] enforces, on each variable-value pair, either AC or some local consistency (LC) property strictly stronger than AC depending on the value of the parameter $p$. The idea is to enforce LC only on the variable-value pairs with few supports, approximated with the rank $(<p)$ of the first found $\mathrm{AC}$-support. We focus on the constraint-based version, $p c$-LC, where $\left\langle x_{i}, v_{i}\right\rangle$ is $p c$-LC if for every constraint $c_{j} \in \operatorname{cons}\left(x_{i}\right),\left\langle x_{i}, v_{i}\right\rangle$ is $p$-stable for $\mathrm{AC}$ on $c_{j}$ or $\left\langle x_{i}, v_{i}\right\rangle$ is $\mathrm{LC}$ on $c_{j}$. In $p c$-LC, the value of $p$ is given as input. In the adaptive version, apc-LC, it is dynamically determined for each constraint $c_{j}$ using the weight of $c_{j}, w\left(c_{j}\right)$, which is the number of times $c_{j}$ caused a domain wipe-out like in the variable-ordering heuristic dom/wdeg [5]: 


$$
p\left(c_{j}\right)=\frac{w\left(c_{j}\right)-\min _{c_{k} \in \mathcal{C}}\left(w\left(c_{k}\right)\right)}{\max _{c_{k} \in \mathcal{C}}\left(w\left(c_{k}\right)\right)-\min _{c_{k} \in \mathcal{C}}\left(w\left(c_{k}\right)\right)+1} .
$$

In [1], apc-maxRPC was experimentally shown to outperform AC and maxRPC [6].

\section{Modifying apc-LC for Non-Binary CSPs}

For binary CSPs, $p$-stability for $\mathrm{AC}$ of $\left\langle x_{i}, v_{i}\right\rangle$ estimates how many supports are left for $\left\langle x_{i}, v_{i}\right\rangle$ in other constraints using the rank of the AC-support in the corresponding domain. This estimate should not directly applied to non-binary table constraints because the GAC-support of $\left\langle x_{i}, v_{i}\right\rangle$ is a tuple in a relation that is unsorted, which would make the estimate way too imprecise. Consider the example with $\left\langle x_{i}, v_{i}\right\rangle$ and a relation $R_{j}$ of 100 tuples. Assume that the only tuple $\tau \in R_{j}$ supporting $\left\langle x_{i}, v_{i}\right\rangle$ appears at the top of the table of $R_{j}$. The estimate would indicate that there are many supports for $\left\langle x_{i}, v_{i}\right\rangle$ because there are 99 tuples that appear after it. However, in reality, $\left\langle x_{i}, v_{i}\right\rangle$ has a unique support. Below, we introduce $p$-stability for GAC, which counts the number of supports for each variable-value pair. Then, we introduce a mechanism to compute $p$-stability for GAC, and finally give an algorithm for enforcing apcLC, which adaptively enforces STR or LC. In this paper, we study $\mathrm{R}(*, 2) \mathrm{C}$ as $\mathrm{LC}$, and discuss the implementation of $\operatorname{apc}-\mathrm{R}(*, 2) \mathrm{C}$.

\section{$3.1 \quad p$-stability for GAC}

We say that $\left\langle x_{i}, v_{i}\right\rangle$ is $p$-stable for $G A C$ if for every constraint $c_{j} \in \operatorname{cons}\left(x_{i}\right)$,

$$
\frac{\left|\sigma_{x_{i}=v_{i}}\left(R_{j}\right)\right|}{\left|R_{j}^{o}\right|} \geq p\left(c_{j}\right)
$$

where $\sigma_{x_{i}=v_{i}}\left(R_{j}\right)$ selects the tuples in $R_{j}$ where $\left\langle x_{i}, v_{i}\right\rangle$ appears, and $R_{j}^{o}$ is the original, unfiltered relation. A CSP is $p$-stable for GAC if every variable-value pair is $p$-stable for GAC for every constraint that applies to it.

Figure 2 gives the relation for the constraint $x_{1} \leq x_{2} .\left\langle x_{1}, 1\right\rangle$ and $\left\langle x_{1}, 2\right\rangle$ are 0.25 -stable for GAC. Indeed, $\sigma_{x_{1}=1}$ returns four rows $\{0,1,2,3\}$ in the table, and $\left\langle x_{1}, 1\right\rangle$ is 0.25 -stable: $\frac{4}{10} \geq 0.25$. Similarly, $\left\langle x_{1}, 2\right\rangle$ also is 0.25 -stable: $\frac{3}{10} \geq 0.25$. $\left\langle x_{1}, 3\right\rangle$ and $\left\langle x_{1}, 4\right\rangle$ are not 0.25 -stable, because $\frac{2}{10} \geq 0.25$ and $\frac{1}{10} \geq 0.25$. This example illustrates how, on binary constraints, and for a given $p, p$-stable for AC does not guarantee $p$-stable for GAC. (Recall that $\left\langle x_{1}, 3\right\rangle$ is 0.25 -stable for $\mathrm{AC}$ in Figure 1).

\subsection{Computing $p$-stability for GAC}

For each constraint $c_{j}$, we introduce for every $\left\langle x_{i}, v_{i}\right\rangle$ a set of integers indicating the position of the tuples returned by $\sigma_{x_{i}=v_{i}}\left(R_{j}\right)$, which is similar to the data structure in GAC4 [17]. We denote this table gacSupports $\left[R_{j}\right]\left[\left\langle x_{i}, v_{i}\right\rangle\right]$. The check for $p$-stable can be verified by using $\mid$ gacSupports $\left[R_{j}\right]\left[\left\langle x_{i}, v_{i}\right\rangle\right] \mid$. Figure 2, shows the gacSupports $\left[R_{j}\right]$ for the constraint $x_{1} \leq x_{2}$. For each relation, the space complexity to store each gacSupports $\left[R_{j}\right]$ is $\mathcal{O}(k \cdot t)$, where $k$ is the maximum constraint arity and $t$ is the maximum number of tuples in a relation. The time complexity to generate gacSupports $\left[R_{j}\right]$ is $\mathcal{O}(k \cdot t)$, by iterating through every tuple. 


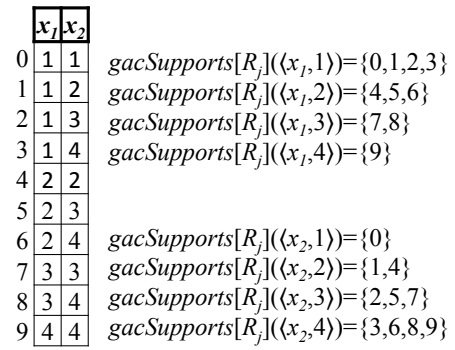

Fig. 2. The relation of $x_{1} \leq x_{2} .\left\langle x_{1}, 3\right\rangle$ and $\left\langle x_{1}, 4\right\rangle$ are not 0.25 -stable for GAC.

\subsection{Algorithm for Enforcing $a p c-L C$}

With the gacSupports data-structure, we can apply STR by verifying, for each constraint $c_{j}$, that every variable $x_{i} \in \operatorname{scope}\left(c_{j}\right)$ and $v_{i} \in \operatorname{dom}\left(x_{i}\right)$ has a nonzero $\mid$ gacSupports $\left[R_{j}\right]\left[\left\langle x_{i}, v_{i}\right\rangle\right] \mid$. Living-STR (Algorithm 1) does precisely this operation (ignoring Lines 4 and 5 , which apply to the apc-LC operation introduced next). past $(\mathcal{P})$ denotes the variables of the CSP $\mathcal{P}$ already instantiated by search, and delTuples $\left(R_{k}, S\right.$, level $)$ deletes all the tuples in the subset $S \subseteq R_{k}$, and marks their removal level at the level of search level. When deleting a tuple from the relation $R_{k}, c_{k}$ 's neighboring constraints, neigh $\left(c_{k}\right)$, should be re-queued to be processed with LIVING-STR. Initially, all constraints are in the queue. LIVING-STR is similar to STR3 in that it iterates over variable-value pairs rather than over tuples. However, it does not use as much book-keeping for optimizing the number of STR checks as STR3 [14]. Instead, LIVING-STR uses the same data structures as STR and STR2(+) to manage tuple deletions in a relation $[13,21]$.

Including Lines 4 and 5 in Algorithm 1 yields apc-LC, which adaptively applies LC. The adaptive level $p\left(c_{j}\right)$ is defined by Balafrej et al. [1] and recalled in Equation (1). The local consistency technique used here is the implementation of $\mathrm{R}(*, 2) \mathrm{C}[12]$, apc- $\mathrm{R}(*, 2) \mathrm{C}$. AppLY- $\mathrm{R}(*, 2) \mathrm{C}$ (Algorithm 2 ) takes as input the list of tuples of a constraint on which $\mathrm{R}(*, 2) \mathrm{C}$ must be enforced. SEArchSup$\operatorname{PORT}\left(R_{i}, \tau,\left\{R_{j}\right\}\right)$ on Line 3 of Algorithm 2 searches for a support for the tuple $\tau \in R_{i}$, the pairwise check [12].

Theoretical analysis: Let $k$ be the maximum constraint arity, $d$ the maximum domain size, and $\delta$ the maximum number of neighbors of a constraint. The time complexity of Algorithm 1 is $\mathcal{O}(k \cdot d)$. Algorithm 2 is $\mathcal{O}\left(\delta \cdot t^{2}\right)$ because it makes $\mathcal{O}(\delta \cdot t)$ calls to SEARCHSUpport, which is $\mathcal{O}(t)$ in our context. The correctness of Algorithms 1 and 2 can be shown in straightforward manner by contradiction.

\section{Empirical Evaluations}

The goal of our experimental analysis is to assess if $a p c-\mathrm{R}(*, 2) \mathrm{C}$ effectively selects when to apply STR and $\mathrm{R}(*, 2) \mathrm{C}$ when used in a pre-processing step and in a real full lookahead strategy [9] during backtrack search to find the first solution to a CSP. In our experiments, we use the variable ordering dom/wdeg [5]. The 

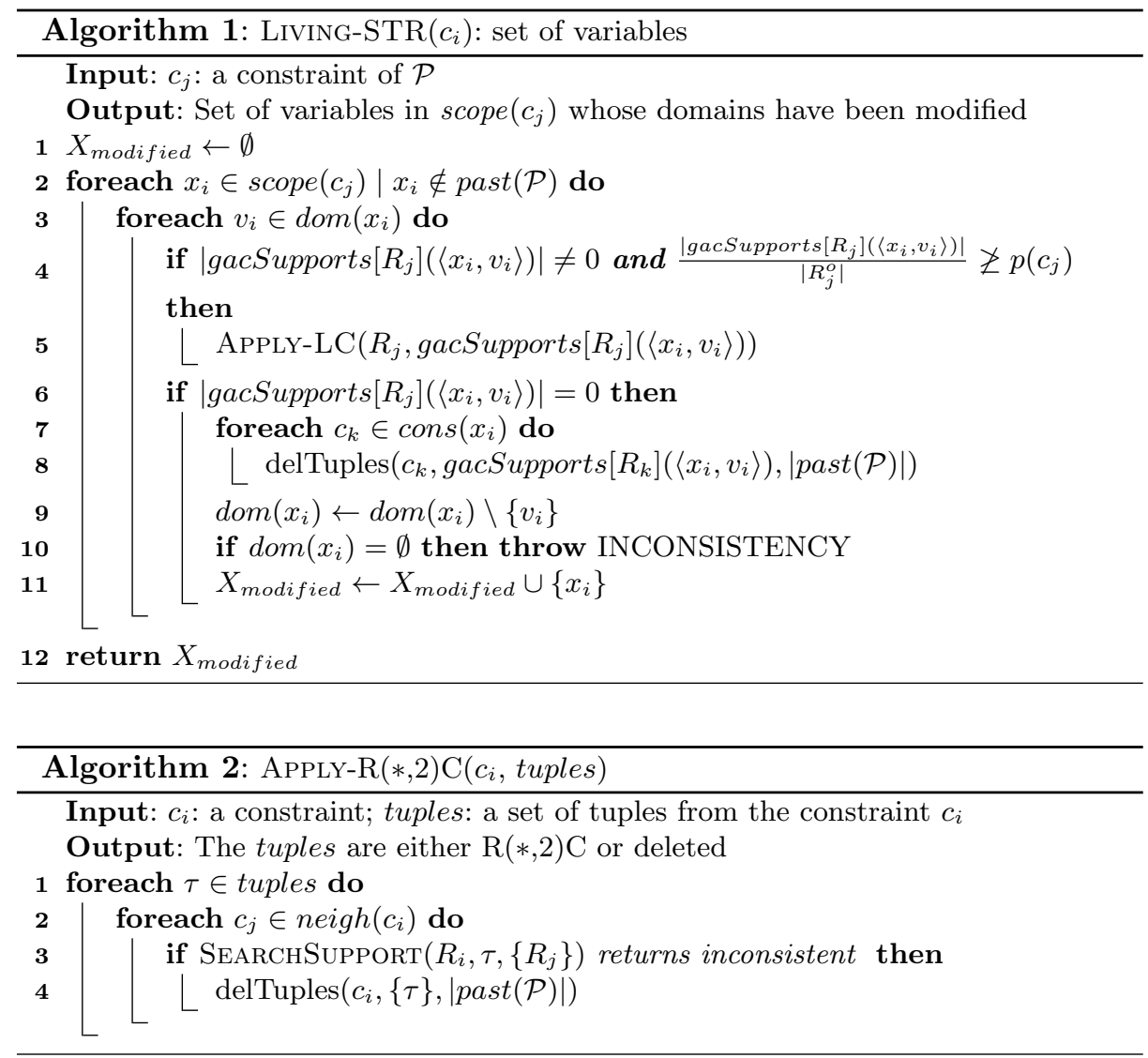

experiments are conducted on the benchmarks of the CSP Solver Competition ${ }^{3}$ with a time limit of two hours per instance and 8 GB of memory. Because STR and $\mathrm{R}(*, 2) \mathrm{C}$ enforce the same level of consistency on binary CSPs [4], we focus our experiments on 21 non-binary benchmarks ${ }^{4}$ consisting of 623 CSP instances. We chose these benchmarks because they are given in extension and at least one algorithm completed $5 \%$ of the instances in the benchmark.

Table 1 summarizes the results in terms of number of instances solved. Importantly, apc- $\mathrm{R}(*, 2) \mathrm{C}$ completes the largest number of instances (552). Considering the instances solved by all algorithms (485 instances), apc- $\mathrm{R}(*, 2) \mathrm{C}$ has the smallest average and median CPU time. Row 3 indicates the number of instances STR solved but $\mathrm{R}(*, 2) \mathrm{C}$ and $a p c-\mathrm{R}(*, 2) \mathrm{C}$ did not solve $(18$ and 11 instances, respectively), thus showing that $a p c-\mathrm{R}(*, 2) \mathrm{C}$, although it may have en-

\footnotetext{
3 http://www .cril.univ-artois.fr/CPAI08/

4 aim-(50,100,200), allIntervalSeries, dag-rand, dubois, jnh(Sat/Unsat), lexVg, modifiedRenault, pret, rand-10-20-10, rand-3-20-20(-fcd), rand-8-20-5, ssa, travellingSalesman-20, travellingSalesman-25, ukVg, varDimacs, wordsVg
} 
Table 1. Number of instances completed by the tested algorithms.

\begin{tabular}{|l|r|r|r|r|}
\cline { 3 - 4 } & STR & $\mathrm{R}(*, 2) \mathrm{C}$ & apc-R $(*, 2) \mathrm{C}$ \\
\hline \hline 1 & \#instances completed by & 504 & 550 & $\mathbf{5 5 2}$ \\
\hline 2 & \#instances completed only by & 10 & 5 & 0 \\
\hline \hline 3 & \#instances solved by STR, but missed by & 0 & 18 & 11 \\
\hline 4 & \#instances solved by R(*,2)C, but missed by & 64 & 0 & 6 \\
\hline 5 & \#instances solved by apc-R(*,2)C, but missed by & 59 & 8 & 0 \\
\hline \hline & Average CPU time (sec.) over 458 instances & 328.41 & 378.12 & $\mathbf{3 1 3 . 3 1}$ \\
\hline & Median CPU time (sec.) over 458 instances & 7.23 & 17.35 & $\mathbf{7 . 2 1}$ \\
\hline
\end{tabular}

forced $\mathrm{R}(*, 2) \mathrm{C}$ too often, outperformed $\mathrm{R}(*, 2) \mathrm{C}$ and missed fewer instances than it (11 vs. 18). Row 4 exhibits similar results showing the number of instances that $\mathrm{R}(*, 2) \mathrm{C}$ could solve, but that were missed by STR and apc- $\mathrm{R}(*, 2) \mathrm{C}(64$ and 6 instances, respectively). Here, apc- $\mathrm{R}(*, 2) \mathrm{C}$ did not enforce $\mathrm{R}(*, 2) \mathrm{C}$ often enough, but managed to outperform STR missing significantly fewer instances than STR (6 vs. 64$)$.

Table 2 gives a finer analysis of the data, showing the number of completions and average and median CPU time per benchmark. Averages computed over only the instances completed by all techniques are shown in the column All. We split

Table 2. Results of the experiments per benchmark, organized in four categories.

\begin{tabular}{|c|c|c|c|c|c|c|c|c|c|c|c|}
\hline Benchmark & 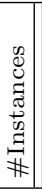 & $\begin{array}{l}\tilde{\mu} \\
\tilde{H} \\
\tilde{\Omega}\end{array}$ & 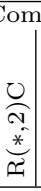 & 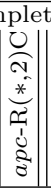 & $\overline{4}$ & Average & 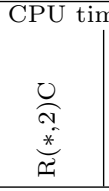 & 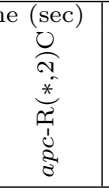 & Median & \multicolumn{2}{|c|}{ 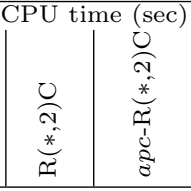 } \\
\hline \multicolumn{12}{|c|}{ a) $a p c-\mathrm{R}(*, 2) \mathrm{C}$ is the best } \\
\hline aim-50 & 24 & 24 & 24 & 24 & 24 & 0.04 & 0.07 & 0.04 & 0.02 & 0.04 & 0.03 \\
\hline allIntervalSeries & 25 & 22 & 22 & 22 & 22 & \begin{tabular}{l|l}
7.09 \\
\end{tabular} & 141.85 & 6.00 & 0.13 & 0.31 & 0.12 \\
\hline jnhSat & 16 & 16 & 16 & 16 & 16 & 13.07 & 357.66 & $\mathbf{1 1 . 7 4}$ & 8.15 & 142.24 & 7.21 \\
\hline modifiedRenault & 50 & 50 & 50 & 50 & 50 & 6.39 & 11.17 & 6.29 & 7.24 & 8.79 & 6.98 \\
\hline$-3-20-20$ & 50 & 31 & 43 & 41 & 31 & \begin{tabular}{|l|l|}
$1,666.10$ \\
\end{tabular} & 939.88 & 932.77 & $1,211.50$ & 822.54 & 811.74 \\
\hline \multicolumn{12}{|c|}{ b) apc- $\mathrm{R}(*, 2) \mathrm{C}$ is competitive } \\
\hline aim- & 24 & 24 & 24 & 24 & 24 & 0.38 & 0.26 & 0.41 & 0.18 & 0.25 & 0.16 \\
\hline aim-200 & 24 & 22 & 24 & 24 & 22 & 414.48 & 6.52 & 286.27 & 2.39 & 1.37 & 2.60 \\
\hline jnhUnsat & 34 & 34 & 34 & 34 & 34 & 13.61 & 294.77 & 13.95 & 10.74 & 153.50 & 9.78 \\
\hline lexVg & 63 & 63 & 63 & \begin{tabular}{|l|l}
63 \\
\end{tabular} & 63 & 69.81 & 341.87 & 338.74 & 0.50 & 1.38 & 0.89 \\
\hline pret & 8 & 4 & 4 & 4 & 4 & 117.89 & 347.03 & 136.04 & 115.81 & 354.82 & 145.70 \\
\hline rand-3-20-20-fcd & 50 & 39 & 48 & 47 & 39 & 928.06 & 546.84 & 615.23 & 501.30 & 422.24 & 464.00 \\
\hline rand-8-20-5 & 20 & 9 & 20 & 20 & 9 & $2,564.94$ & 355.57 & 372.76 & $1,987.35$ & 314.26 & 261.68 \\
\hline rand-10-20-10 & 20 & 12 & 12 & 12 & 12 & 6.72 & 1.67 & 2.76 & 6.40 & 1.66 & 2.75 \\
\hline ssa & 8 & 6 & 5 & \begin{tabular}{l|l}
6 \\
\end{tabular} & 5 & 64.60 & 100.64 & 69.59 & 1.51 & 1.60 & 1.58 \\
\hline TSP-25 & 15 & 13 & 10 & 13 & 10 & 232.38 & $1,072.72$ & 743.33 & 69.00 & 211.41 & 131.69 \\
\hline $\mathrm{ukVg}$ & 65 & 37 & 31 & 34 & 31 & 166.82 & 796.90 & 421.35 & 36.29 & 54.65 & 30.39 \\
\hline varDimacs & 9 & 6 & 6 & 6 & 6 & 89.23 & 587.55 & 319.20 & 1.56 & 6.43 & 2.94 \\
\hline wordsVg & 65 & 65 & 58 & 58 & 58 & 119.76 & 532.05 & 400.22 & 0.39 & 0.95 & 0.59 \\
\hline \multicolumn{12}{|c|}{ c) apc- $\mathrm{R}(*, 2) \mathrm{C}$ is the worst } \\
\hline dubois & 13 & 7 & 8 & \begin{tabular}{l|l}
6 \\
\end{tabular} & 6 & $1,000.54$ & 451.91 & $1,456.01$ & 552.13 & 255.25 & 779.57 \\
\hline TSP-20 & 15 & 15 & 15 & \begin{tabular}{|l||}
15 \\
\end{tabular} & 15 & \begin{tabular}{|l|l|}
101.20 \\
\end{tabular} & 318.37 & \begin{tabular}{l|l|}
335.13 \\
\end{tabular} & 23.32 & 61.55 & 46.34 \\
\hline \multicolumn{12}{|c|}{ d) Not solved by STR } \\
\hline dag-rand & $25 \mid$ & 0 & 25 & 25 & 0 & - & 123.70 & 149.64 & & 124.47 & 151.33 \\
\hline
\end{tabular}


the table into four categories based on the average $\mathrm{CPU}$ time of apc-R $(*, 2) \mathrm{C}$ : a) apc- $\mathrm{R}(*, 2) \mathrm{C}$ performs the best (5 benchmarks); b) apc- $\mathrm{R}(*, 2) \mathrm{C}$ is competitive, performing between STR and $\mathrm{R}(*, 2) \mathrm{C}(13$ benchmarks); c) apc- $\mathrm{R}(*, 2) \mathrm{C}$ performs the worst (2 benchmarks); and $d$ ) STR does not solve the benchmark but $\mathrm{R}(*, 2) \mathrm{C}$ and apc-R $(*, 2) \mathrm{C}$ do (1 benchmark). The best average CPU time appears in bold face in the corresponding column. The median CPU time of apc- $\mathrm{R}(*, 2) \mathrm{C}$ is bold faced when its rank differs from that of the average CPU time (on which the four categorized are based). On TSP-20, apc-R $(*, 2) \mathrm{C}$ ranks bottom on average CPU time but between STR and $\mathrm{R}(*, 2) \mathrm{C}$ on median $\mathrm{CPU}$ time. On aim-100, jnhUnsat, rand-8-20-5, and $\mathrm{ukVg}$, apc- $\mathrm{R}(*, 2) \mathrm{C}$ is between $\mathrm{STR}$ and $\mathrm{R}(*, 2) \mathrm{C}$ for average $\mathrm{CPU}$ time, but best for median CPU time.

Table 3 shows the average number of STR and $\mathrm{R}(*, 2) \mathrm{C}$ checks that apc$\mathrm{R}(*, 2) \mathrm{C}$ performs per benchmark. In allIntervalSeries, no calls are made to

Table 3. Number of calls to STR and $\mathrm{R}(*, 2) \mathrm{C}$ by benchmark.

\begin{tabular}{|c|c|c|c|c|c|}
\hline Benchmark & STR checks & $\mathrm{R}(*, 2) \mathrm{C}$ checks & Benchmark & STR checks & $\mathrm{R}(*, 2) \mathrm{C}$ checks \\
\hline \multicolumn{3}{|c|}{ a) $a p c-\mathrm{R}(*, 2) \mathrm{C}$ is the best } & \multicolumn{3}{|c|}{ b) apc- $\mathrm{R}(*, 2) \mathrm{C}$ is competitive } \\
\hline aim-50 & 456,823 & 39,491 & aim-100 & $7,731,585$ & 894,353 \\
\hline allIntervalSeries & $38,281,694$ & 0 & aim-200 & $1,160,334,482$ & $163,177,907$ \\
\hline jnhSat & $22,119,135$ & 599,080 & jnhUnsat & $51,688,166$ & $1,918,781$ \\
\hline modifiedRenault & $4,618,778$ & 601,641 & lexVg & $564,010,457$ & $2,180,503,026$ \\
\hline \multirow[t]{2}{*}{ rand-3-20-20 } & $489,441,126$ & $3,480,216,943$ & pret & $422,987,946$ & $13,973,748$ \\
\hline & & & rand-3-20-20-fcd & $455,664,100$ & $2,956,467,994$ \\
\hline \multirow{5}{*}{$\begin{array}{c}c) a p c-1 \\
\text { dubois } \\
\text { TSP-20 }\end{array}$} & $2(*, 2) \mathrm{C}$ is the & rst & rand-8-20-5 & $77,470,561$ & $184,764,543$ \\
\hline & $3,343,830,604$ & $4,668,288$ & rand- $10-20-10$ & 72,608 & 3,972 \\
\hline & $622,949,698$ & $991,590,957$ & ssa & $156,631,370$ & $11,689,961$ \\
\hline & & & TSP-25 & $2,903,953,315$ & $3,947,391,769$ \\
\hline & & & $\mathrm{ukVg}$ & $341,565,892$ & $1,002,334,753$ \\
\hline \multicolumn{3}{|c|}{ d) Not solved by STR } & varDimacs & $720,843,958$ & $84,123,204$ \\
\hline dag-rand & 359,248 & 21,870 & wordsVg & $514,840,737$ & $2,052,367,934$ \\
\hline
\end{tabular}

$\mathrm{R}(*, 2) \mathrm{C}$ because the instance is solved backtrack free with STR alone. For apc$\mathrm{LC}$, no call to $L C$ is done during pre-processing because the weights of all the constraints are set to 1 (giving $p\left(c_{j}\right)=0$ for all $c_{j} \in \mathcal{C}$ ) and updated only during search. For dag-rand, there is a smaller number of $\mathrm{R}(*, 2) \mathrm{C}$ calls than STR calls $(21,870$ vs. 359,248$)$. However, those few calls allow us to solve all the instances of this benchmark whereas STR alone could not solve any instance. This result is a glowing testimony of the ability of $a p c-\mathrm{R}(*, 2) \mathrm{C}$ to apply the appropriate level of consistency where needed.

\section{Conclusions}

In this paper, we extend the notion of $p$-stability for $\mathrm{AC}$ to GAC, and provide a mechanism for computing it. We give an algorithm for enforcing apc- $\mathrm{R}(*, 2) \mathrm{C}$ on non-binary table constraints, which adaptively enforces GAC and $\mathrm{R}(*, 2) \mathrm{C}$. We validate our approach on benchmark problems. Future work is to investigate other adaptive criteria for selecting the level of consistency to apply, in particular one that operates during both pre-processing and search. To apply our approach to constraints defined in intension and other global constraints, we could use techniques that approximate the number of solutions in those constraints [19]. 


\section{References}

1. Balafrej, A., Bessiere, C., Coletta, R., Bouyakhf, E.H.: Adaptive Parameterized Consistency. In: Proc. CP 2013. LNCS, vol. 8124, pp. 143-158. Springer (2013)

2. Bessiere, C.: Handbook of Constraint Programming, chap. Constraint Propagation, pp. 29-83. Elsevier (2006)

3. Bessière, C., Régin, J.C., Yap, R.H., Zhang, Y.: An Optimal Coarse-Grained Arc Consistency Algorithm. Artificial Intelligence 165(2), 165-185 (2005)

4. Bessière, C., Stergiou, K., Walsh, T.: Domain Filtering Consistencies for NonBinary Constraints. Artificial Intelligence 172, 800-822 (2008)

5. Boussemart, F., Hemery, F., Lecoutre, C., Sais, L.: Boosting Systematic Search by Weighting Constraints. In: Proc. ECAI 2004. pp. 146-150 (2004)

6. Debruyne, R., Bessière, C.: From Restricted Path Consistency to Max-Restricted Path Consistency. In: Proc. CP 97. LNCS, vol. 1330, pp. 312-326. Springer (1997)

7. Geschwender, D., Karakashian, S., Woodward, R., Choueiry, B.Y., Scott, S.D.: Selecting the Appropriate Consistency Algorithm for CSPs Using Machine Learning Techniques. In: Proc. of AAAI 2013. pp. 1611-1612 (2013)

8. Gyssens, M.: On the Complexity of Join Dependencies. ACM Trans. Database Systems 11(1), 81-108 (1986)

9. Haralick, R.M., Elliott, G.L.: Increasing Tree Search Efficiency for Constraint Satisfaction Problems. Artificial Intelligence 14, 263-313 (1980)

10. Janssen, P., Jégou, P., Nougier, B., Vilarem, M.C.: A Filtering Process for General Constraint-Satisfaction Problems: Achieving Pairwise-Consistency Using an Associated Binary Representation. In: IEEE Workshop on Tools for AI. pp. 420-427 (1989)

11. Kadioglu, S., Malitsky, Y., Sabharwal, A., Samulowitz, H., Sellmann, M.: Algorithm Selection and Scheduling. In: Proc. CP 2011. LNCS, vol. 6876, pp. 454-469. Springer (2011)

12. Karakashian, S., Woodward, R., Reeson, C., Choueiry, B.Y., Bessiere, C.: A First Practical Algorithm for High Levels of Relational Consistency. In: Proc. AAAI 2010. pp. 101-107 (2010)

13. Lecoutre, C.: STR2: Optimized Simple Tabular Reduction for Table Constraints. Constraints 16(4), 341-371 (2011)

14. Lecoutre, C., Likitvivatanavong, C., Yap, R.H.C.: A Path-Optimal GAC Algorithm for Table Constraints. In: Proc. of ECAI 2012. pp. 510-515 (2012)

15. Lecoutre, C., Paparrizou, A., Stergiou, K.: Extending STR to a Higher-Order Consistency. In: Proc. AAAI 2013. pp. 576-582. Bellevue, WA (2013)

16. Mackworth, A.K.: Consistency in Networks of Relations. AI 8, 99-118 (1977)

17. Mohr, R., Masini, G.: Good Old Discrete Relaxation. In: European Conference on Artificial Intelligence (ECAI-88). pp. 651-656. Munich, W. Germany (1988)

18. Paparrizou, A., Stergiou, K.: Evaluating Simple Fully Automated Heuristics for Adaptive Constraint Propagation. In: Proc. of ICTAI 2012. pp. 880-885 (2012)

19. Pesant, G., Quimper, C.G., Zanarini, A.: Counting-Based Search: Branching Heuristics for Constraint Satisfaction Problems. JAIR 43, 173-210 (2012)

20. Stergiou, K.: Heuristics for Dynamically Adapting Propagation. In: Proc. of ECAI 2008. pp. 485-489 (2008)

21. Ullmann, J.R.: Partition Search for Non-binary Constraint Satisfaction. Information Sciences 177(18), 3639-3678 (Sep 2007)

22. Xu, L., Hutter, F., Hoos, H.H., Leyton-Brown, K.: SATzilla: Portfolio-Based Algorithm Selection for SAT. JAIR 32, 565-606 (2008) 